\section{GW23-e0993 ABCG1 PLAYS AN IMPORTANT ROLE IN KEEPING ENDOTHELIAL FUNCTION ASSOCIATED WITH ENDOPLASMIC RETICULUM STRESS}

doi:10.1136/heartjnl-2012-302920a.26

Xuejiahong Xuejiahong. Department of Cardiovascular Medicine, Second Affiliated Hospital, Xi'an Jiaotong University College of Medicine, Xi'an 710004, China

Objectives ATP binding cassette (ABC) transporters play an important role in regulating sterol homeostasis in many cell types. Endothelial cells (EC) have a high capacity to efflux sterols and express the $A B C$ transporter $A B C G 1$. It is reported that $A B C G 1$ deficiency increases inflammatory responses in vascular $\mathrm{EC}$ and intracellular free cholesterol accumulation can activate endoplasmic reticulum stress (ERS). Here, the study was objected to observe the relationship between ABCG1 and endothelial protection and concerned mechanism of ERS.

Methods Recombinant ABCG1 overexpression plasmid and siRNA ABCG1 were transfected into human umbilical vein endothelial cells (HUVECs). Real-time PCR and Western blot were explored to exam ABCG1 expression. The rate of cholesterol efflux to HDL was measured by scintillation counting, and intracellular lipid content was measured by enzymatic fluorometric method. Endothelial activation was measured by eNOS expression and NO activity. In addition, the level of IL- 6 and TNF $\alpha$ were measured by ELISA. GRP78 and CHOP protein expression were used to show activated ERS.

Results Using siRNA, we obtained a significant $70 \%$ reduction in ABCG1 mRNA expression in HUVECs. Compared with normal controls, downregulation of ABCG1 by siRNA decreased eNOS protein expression by $50 \%$ and $\mathrm{NO}$ activity by $30 \%$ accompanied with decreased intracellular cholesterol efflux in HUVECs. Moreover, levels of IL- 6 and TNF $\alpha$ in ABCG1 siRNA endothelial cells were increased by 3 and 4 times respectively. Similarly, GRP78 and CHOP protein expression were significantly increased 3 and 4 times in ABCG1 siRNA endothelial cells. On the contrary, upregulation of ABCG1 using recombinant overexperssion plasmid decreased GRP78 and $\mathrm{CHOP}$ protein expression and conversely increased eNOS protein expression by $20 \%$ and $\mathrm{NO}$ activity by $20 \%$.

Conclusions There appears to be a critical link between ABCG1 expression and endothelial function in vascular EC. ABCG1 deficiency promotes endothelial activity that seems to be associated with activated ERS. Upregulation of ABCG1 has an effective effect on protection of endothelial function. 